\title{
CFD Analysis of Bifurcated Human Carotid Artery: Newtonian Approach
}

\author{
Md. Syamul Bashar, Rifat Hossain, Ranju Singha Bittu, Md. Shafiqul Islam \\ Department of Mechanical Engineering \\ Shahjalal University of Science and Technology (SUST), \\ Sylhet, Bangladesh
}

\begin{abstract}
Cardiovascular system of human body is an important network since it provides ingredients like oxygen, carbon di oxide, hormones etc. to the individual cells throughout the human body. In the current study, a bifurcated human carotid artery is our point of interest. Blood has been considered as Newtonian fluid in the simulation. Finite Volume Method (FVM) based on Navier-Stokes equation is used to analyze the flow inside the artery. Ansys-FLUENT is used to conduct numerous simulations to find out different effects on bifurcated artery. Three models based on different bifurcation angle have been developed. Velocity contour at different timescale of a cardiac cycle has been studied. All three models have been used to find out the velocity distribution at SS3 and EC2 planes. The pressure distribution has also been studied as well. Wall shear stress (WSS) for Model 2 has been analyzed. For all the simulations 70:30 flow division has been used. Hence, $70 \%$ of the total blood flows through the Internal Carotid Artery (ICA) and $30 \%$ flow occurs through the External Carotid Artery (ECA).
\end{abstract}

Keywords-Carotid artery; wall shear stress; bifurcation; Newtonian

\section{INTRODUCTION}

The very first part of the arterial tree is aorta and its function is to carry blood from left ventricle. This aorta then gets branched and bifurcated into many small arteries and carries blood to different parts of the body. These branches can be categorized in many divisions. Some most common and important are as follows:

- Subclavian Artery (SA): supply blood to upper arms.

- Carotid Artery (CA): supply blood to the brain, head, face.

- Iliac Artery (IA): that supply blood to legs.

The diameter of daughter arteries is usually less than the diameter of parent artery $[1,2]$. The branching of the artery i.e. the bifurcation angle normally varies with site to site and the range can be 10 degrees to 90 degrees. Since the beginning of the $15^{\text {th }}$ century, there are a lot of noteworthy and illustrative works on vascular system and anatomy. Bharadvaj et al. [3] performed some physical experiments to observe the velocity and pressure distribution along the bifurcation of the arteries. Ku and Giddens [4] and Motomiya and Karino [5] also performed similar experiments. The results obtained from the above experiments have been incorporated to justify numerical solutions by Fernandez et al. [6] and Rindt et al. [7]. Perktold et al. [8] considered three-dimensional and pulsatile flow using a Finite Element method and observed the effects of Newtonian and non-Newtonian flows. Regarding the geometry issue, Perktold et al. [9] investigated various bifurcation angles and presented the effects on blood flow due to the changes in the geometric bifurcation angles. The flow of blood through an artery having two consecutive bends and the influence of bend curvature was examined by Hoogstraten et al. [10]. Berger et al. [11] performed similar type of experiments on curved pipes. Since the geometry of the artery is of primary importance in the blood flow, several authors have introduced various bifurcation geometries [12-16].

\section{MEthodology}

Continuity equation and Navier-stokes equation are assigned to describe the blood flow through the carotid artery. These equations were solved using FLUENT (Ansys 15).

Continuity equation: $\nabla \cdot \bar{U}=0, \frac{\partial u}{\partial x}+\frac{\partial v}{\partial y}+\frac{\partial w}{\partial z}$

Navier-stokes equation: $\frac{\partial \bar{U}}{\partial t}+\bar{U} \cdot \nabla \bar{U}=-\frac{\Delta P}{\rho}+\mu \nabla^{2} \bar{U}$

\section{A. Model Construction}

Three different carotid arterial models are investigated under the physiological flow condition. The model geometries are depicted in Fig. 1. The geometrical data of models are derived from Bharadvaj et al., 1982 [17]. The length of common carotid artery (CCA) is taken as $130 \mathrm{~mm}$ as it is the average length of CCA [17]. The cross-sections of the carotid arteries are circular. Bifurcation angle for model 1 and 3 are 40 degree and 60 degree respectively and the angles are symmetrically divided by the CCA axis. And the bifurcation angle for model 2 is constructed in accordance with Bharadvaj et al., 1982 [17]. Geometric models have been constructed using SolidWorks 2017. Different planes on the model arteries are shown in the Fig. 1.

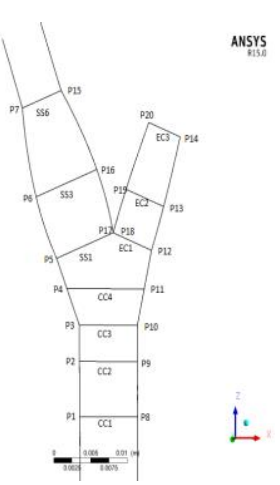

(a) Model 1

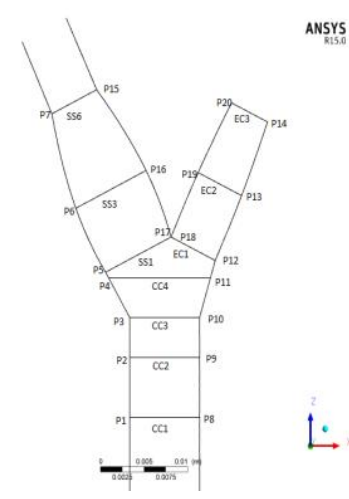

(b) Model 2 


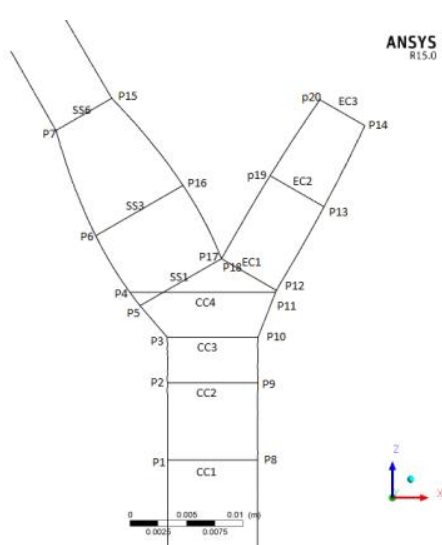

(c) Model 3

Fig. 1. Bifurcation plane of model arteries

\section{B. Grid Indepency Test}

Before conducting to the simulation study, mesh sensitivity tests were performed for the model (Bharadvaj et al., 1982) at uniform steady flow, $\mathrm{u}=0.333 \mathrm{~m} / \mathrm{s}$, and a flow split of 80:20 into internal and external carotid artery. Three different mesh densities were used for mesh sensitivity test from 729035 to 1042713 tetrahedral cells. The adopted number of computational grid cells for model (Bharadvaj et al., 1982) is 848201. Mesh sensitivity tests were also performed for the model 1 , model 2 and model 3 . Tetrahedrons cells are adopted for meshing purpose. Number of nodes and elements are respectively 696328 and 389467 for model 1. For model 2, number of nodes and elements are respectively 735776 and 407191 . And For model 3, number of nodes and elements are respectively 680772 and 379956.

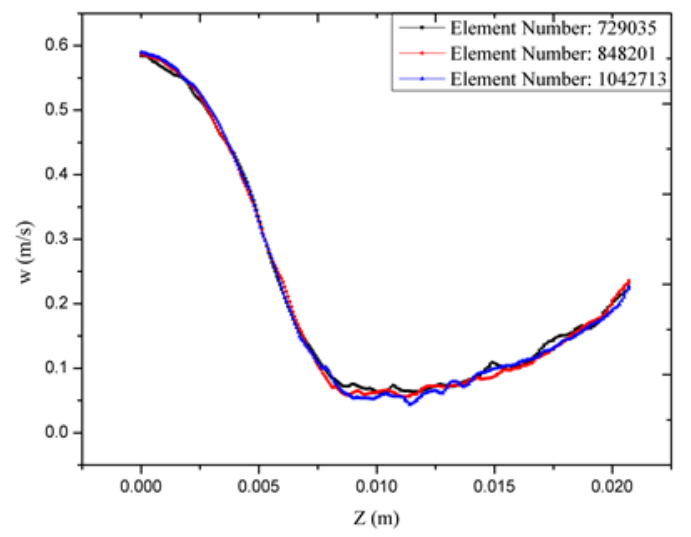

Fig. 2. Axial velocity ' $w$ ' in the plane of bifurcation along ECA axis for the model (Bharadvaj et al., 1982)

\section{Numerical Method}

For the numerical validation of Bharadvaj et. al., 1982, k- $\omega$ SST viscous model has been used. And later in case of pulsatile flow the Large Eddy Simulation (LES) is used as viscous model and bounded second Order Implicit option is used as Transient Formulation.

In the pulsatile simulations, a total of 86 uniform time steps per cardiac cycle were used and the simulation was carried out over six the simulation was based on the residual in continuity falling below a prescribed value $\left(10^{-6}\right.$ for pulsatile flow and $10^{-5}$ for steady flow). Under those conditions, differences in velocities between successive cycles were smaller than $10^{-5}$.

\section{Boundary Condition}

For Newtonian fluid, $\mu$ is taken $0.0035 \mathrm{~kg} / \mathrm{m}-\mathrm{s}$. In the simulation, time varying temporal physiological waveform of velocity was specified at the inlet of the Common carotid Artery (CCA). The peak and mean flows in the CCA were given as $1.107 \mathrm{~m} / \mathrm{s}$ and $0.5067 \mathrm{~m} / \mathrm{s}$, respectively. The period of cardiac cycle is 0.857 second. At the outlet, the outflow boundary conditions were applied to the ICA to the ECA outlet. The walls of carotid artery bifurcation are considered as rigid walls and no-slip boundary conditions are imposed on the walls.

\section{RESULT AND DISCUSSION}

Three-dimensional pulsatile flow in the carotid artery is numerically simulated for models. Six different time locations are considered at sixth cardiac cycle namely, $\mathrm{t} 1=4.40 \mathrm{~s}$ (diastolic flow rate), $\mathrm{t} 2=4.57 \mathrm{~s}$ (minimum flow rate), $\mathrm{t} 3=4.60 \mathrm{~s}$ (accelerating flow), $\mathrm{t} 4=4.63 \mathrm{~s}$ (maximum flow rate), $\mathrm{t} 5=4.88 \mathrm{~s}$ (local minimum flow rate) and $\mathrm{t} 6=4.98 \mathrm{~s}$ (local maximum flow rate). Some velocity contours are shown in Fig. 3.

\section{A. Velocity Distribution Analysis}

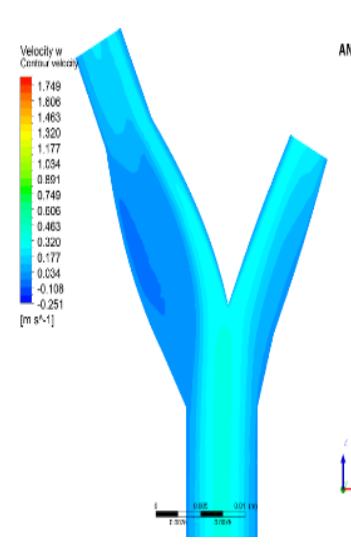

(a) At $\mathrm{t} 2$

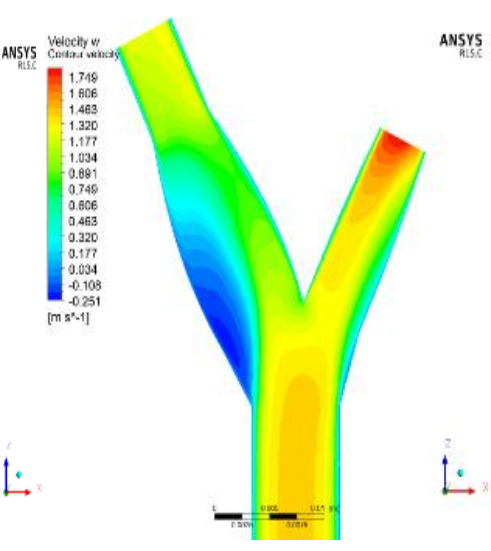

(b) At 4

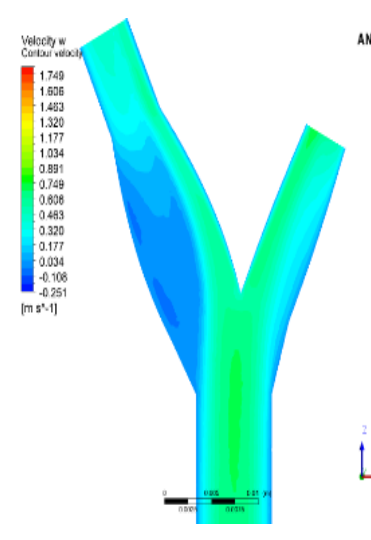

(c) At t5

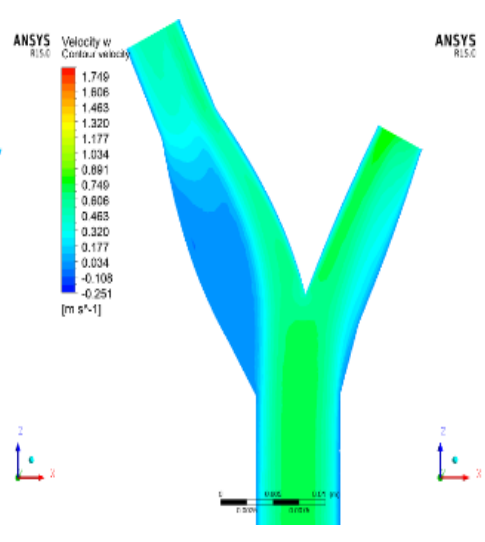

(d) At t6
Fig. 3. Velocity w contours on the bifurcation plane of model 2 for Newtonian case and $70 \%$ flow through ICA. 
B. Velocity Distribution for Different Bifurcation Angle at SS3

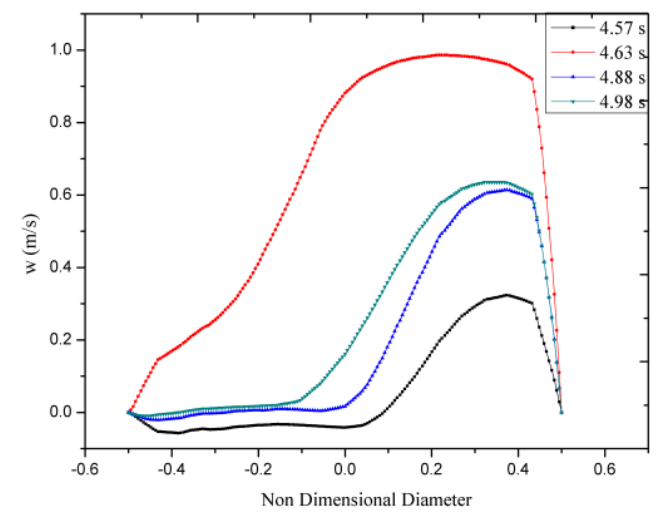

(a) Model 1 (angle 40 degree)

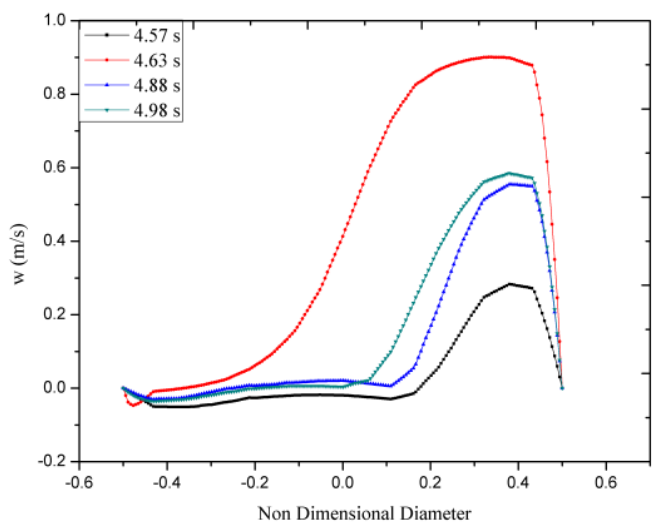

(b) Model 2 (angle 50.5 degree)

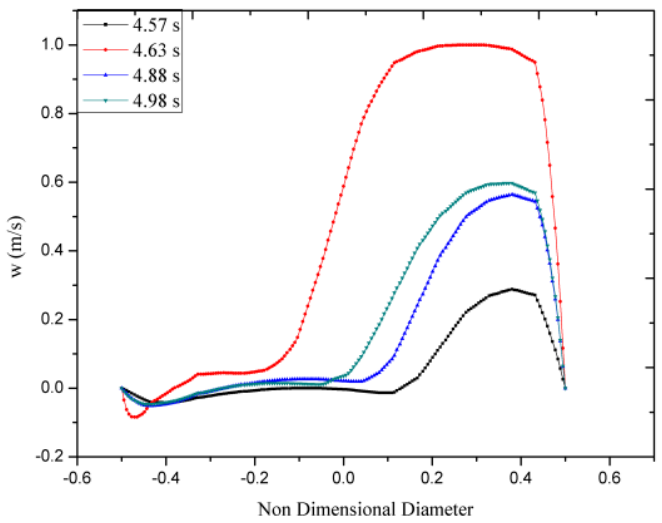

(c) Model 3 (angle 60 degree)

Fig. 4. Axial velocity profiles on the plane of bifurcation at the SS3 for Newtonian flow and $70 \%$ flow through ICA

As volume of flow increases into the ICA, the strong inertia overcomes the adverse pressure gradient occurred in the outer region of the ICA during the maximum blood flows as shown in Fig. 4. Minimum reversed flow occurrs at $t 4$ due to the strong momentum to overcome the secondary flow.
C. Velocity Distribution for Different Bifurcation Angle at EC2

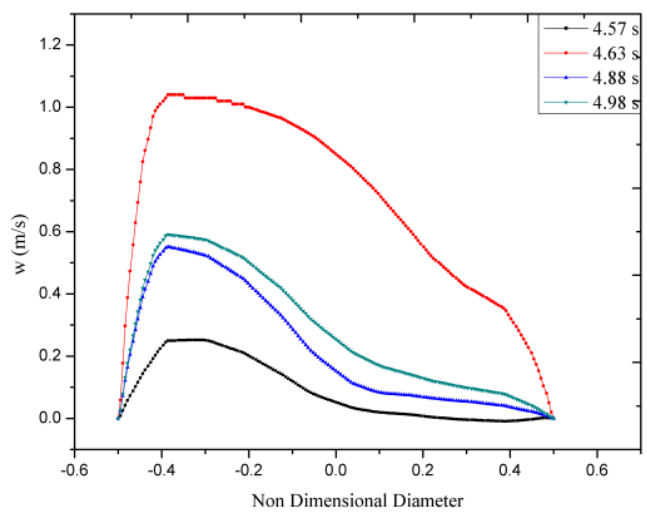

(a) Model 1 (angle 40 degree)

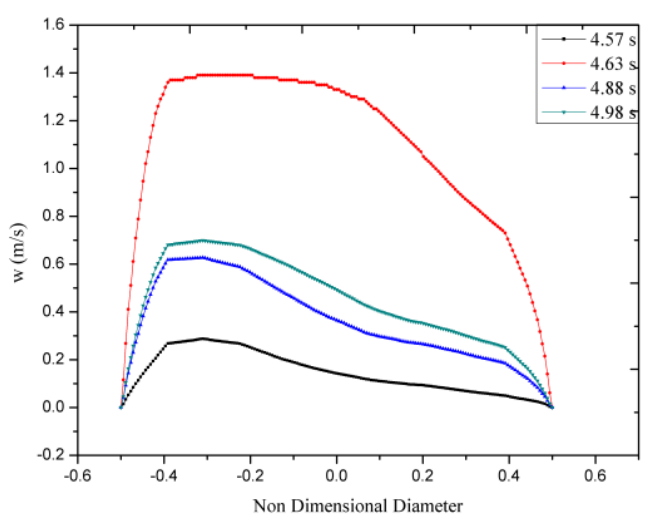

(b) Model 2 (angle 50.5 degree)

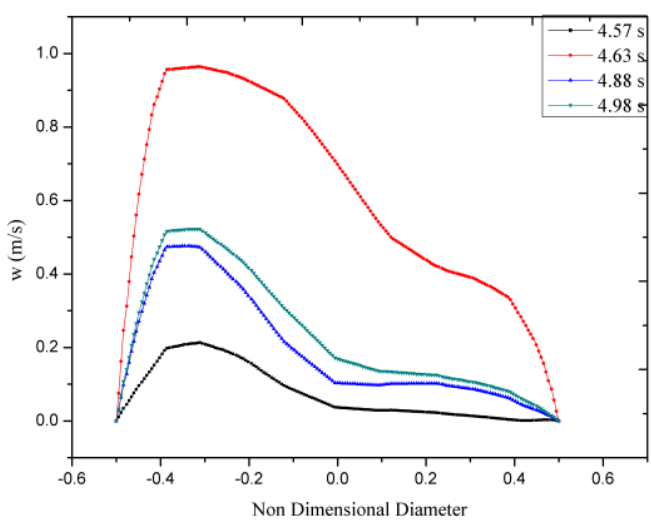

(c) Model 3 (angle 60 degree)

Fig. 5. Axial velocity profiles on the plane of bifurcation at the EC2 for Newtonian flow and $70 \%$ flow through ICA

In the line EC2 no reversed flow occurs. It appears that the peak velocity is skewed toward the inner wall at the both of SS3 and EC2 for all the vessel models as shown in Fig. 4 and Fig. 5. When the flow rate increases into the ICA in Fig. 4, the reverse flow in the outer wall of ICA will recover and reduce the flow recirculation zone due to the strong 
acceleration to overcome the adverse pressure gradient. The flow separation region can be observed at the outer wall due to the adverse pressure gradient as well as the abrupt change in curvature.

\section{Pressure Distrubution Analysis}

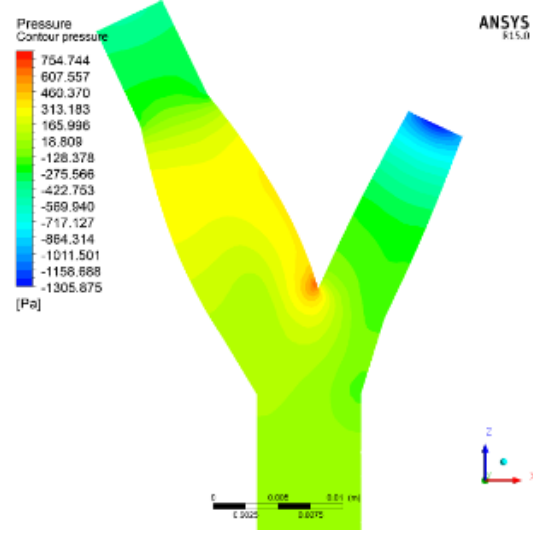

Fig. 6. Pressure contours in the bifurcation plane of model 2 for Newtonian case and $70 \%$ flow through ICA

Form Fig. 6, it is clear that the pressure is the highest at a location where the flow splits into ICA and ECA. For Newtonian case the highest pressure happens towards the ICA inner wall. The overall pressure contour for the Newtonian case shows that higher pressure is generated at the sinus than the ICA and ECA.

\section{E. Wall Shear Stress(WSS) Distribution Analysis}

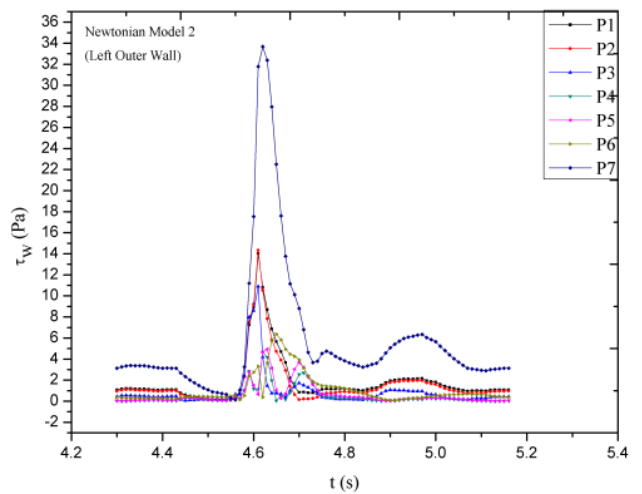

(a) Wall shear stress at left outer wall

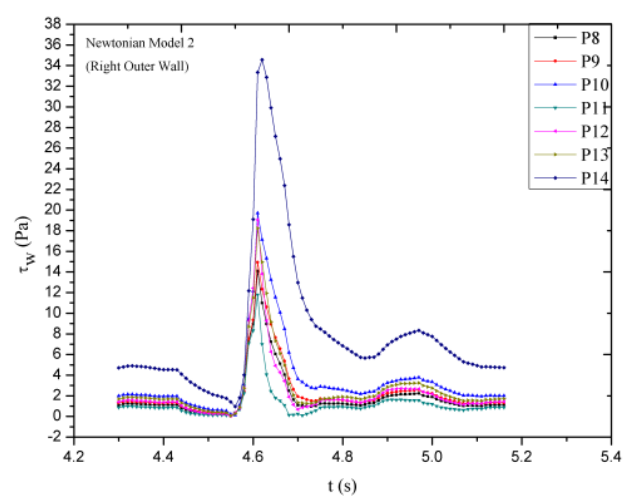

(b) Wall shear stress at right outer wall

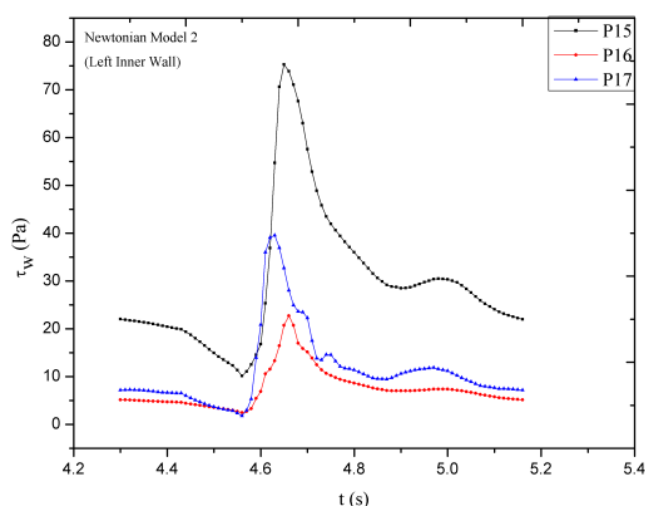

(c) Wall shear stress at left inner wall

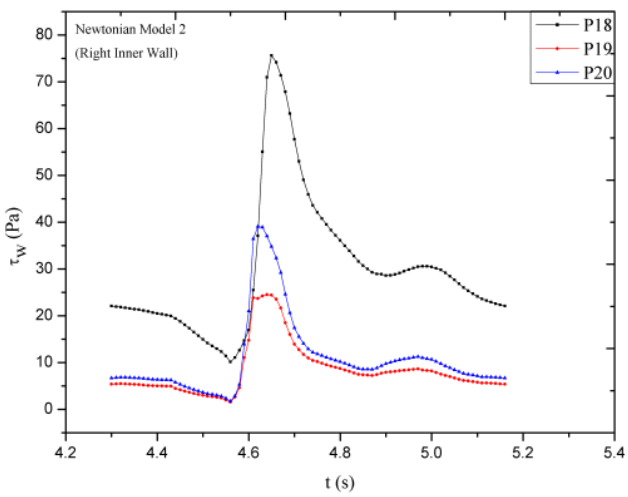

(d) Wall shear stress at right inner wall

Fig. 7. Temporal wall shear stress distributions for Model 2 for flow division 70:30 (70\% flow through ICA)

From Fig. 7, it is visible that the inner wall of the carotid bifurcation in the region where flow division occurs is subjected to relatively high WSS, while the outer wall is the zone of relatively low WSS.

\section{CONCLUSION}

Velocity contours at different times of the sixth cardiac cycle have been visualized. Three models with three different bifurcation angles have been analyzed for velocity distribution in different planes on the artery. The velocity is maximum at time 44 for both SS3 and EC2 planes. The highest pressure for Newtonian case is observed at the bifurcation zone and it is slightly inclined towards the ICA zone. From the WSS distribution of the Model 2, we can conclude the WSS is much higher in the inner wall than the outer wall. Since the models are generated based on the data available from literature, the models may lack patient-specific arteries. Although human blood contains particles such as red blood cell (RBC) and platelets, the blood flow is assumed as a single phase flow. The geometric parameters and inlet blood flow conditions are diverse for each patient. The abovementioned facts can be considered and more insight on this topic can be achieved.

\section{ACKNOWLEDGMENT}

We would like to acknowledge the contribution of everyone who helped us to finish this work. We are thankful 
to the Department of Mechanical Engineering, SUST for providing computational facilities.

\section{REFERENCES}

[1] Sherman, T.F., (1981). "On connecting large vessels to small. The meaning of Murray's law”, The Journal of general physiology, 78(4), pp.431-453.

[2] Adam, J.A., (2011). "Blood vessel branching: beyond the standard calculus problem", Mathematics Magazine, 84(3), pp.196-207.

[3] Bharadvaj, B.K., Mabon, R.F., Giddens, D.P., "St Model of a Human Carotid Bifurcation," Journal of Biomechanics, Vol.15, pp.363378,1982 .

[4] Ku, D.N., and Giddens, D.P., "Pulsatile Flow in a Model Carotid Bifurcation," Arteriosclerosis, Vol.3, 1983.

[5] Motomiya, M., and Karino, T., "Flow Patterns in the Human Carotid Artery Bifurcation," Stroke, Vol.15, 1984.

[6] Fernandez, R.C., DeWitt, K.J., and Botwin, M.R., "Pulsatile Flow through a Bifurcation with Applications to Arterial Disease," Journal of Biomechanics, Vol.9, pp.575-580, 1986.

[7] Rindt, C.C.M., Van de Vosse, F.N., Van Steenhoven, A.A., and Janssen, J.P., "A Numerical and Experimental Analysis of the Flow Field in a Two-Dimensional Model of the Human Carotid Bifurcation," Journal of Biomechanics, Vol.20, pp.499-509,1987.

[8] Perktold, K., Resch, M., and Florian, H., "Pulsatile Non-Newtonian Flow Characteristics in a Three-Dimensional Human Carotid Bifurcation Model," Journal of Biomechanical Engineering, Vol.113, pp.464-475, 1991.
[9] Perktold, K., Peter, R.O., Resch, M., "Pulsatile Non-Newtonian Blood Flow in Three-Dimensional Carotid Bifurcation Models: A Numerical Study of Flow Phenomena under Different Bifurcation Angles," Journal of Biomedical Engineering, Vol. 13, pp.507-515,1991.

[10] Hoogstraten, H.W., Kootstra, J.G., Hillen, B., Krijger, J.K.B., and Wensing, P.J.W., "Numerical Simulation of Blood Flow in an Artery with Two Successive Bends," Journal of Biomechanics, Vol.29, pp.1075-1083, 1996.

[11] Berger, S.A., Talbot, L., and Yao, L.S., "Flow in Curved Pipes," Annu. Rev. Fluid Mechanics, 15, 1983.

[12] Fukushima, T., Homma, T., Harakawa, K., Sakata., N., and Azuma, T., "Vortex Generation in Pulsatile Flow through Arterial Bifurcation Models Including Human Carotid Artery," Journal of Biomechanical Engineering, Vol.110, pp.166-171, 1988.

[13] Yung, C., De Witt, K. J., and Keith, T. G., "Three-Dimensional Steady Flow through a Bifurcation," ASME Journal of Biomechanical Engineering, Vol. 112, pp.189-197, 1990.

[14] Cole, J.S., Gillan, M.A., and Raghunathan, S., "A CFD Study of Steady and Pulsatile Flows Within an Arterial Bifurcation," AIAA-1998-790, 1998.

[15] Kim, C., Kiris, C., and Kwak, D., "Numerical Models of Human Circulatory System under Altered Gravity: Brain Circulation," AIAA2004-1092, 2004.

[16] Gijsen, F.G.H., Van de Vosse, F.N and Janssen, J.D., "The Influence of the Non-Newtonian Properties of Blood on the Flow in Large Arteries: Steady Flow in a Carotid Bifurcation Model," Journal of Biomechanics, Vol.32, pp.601-608, 1999.

[17] B. K. BHARADVAJ, R. F. MABON and D. P. GIDDENS, (1982). "Steady flow in a model of the human carotid bifurcation. Part II-Laser -doppler anemometer measurements". J Biomechanics Vol. 15. No. 5 pp. 363-378. 1982. 